\title{
AN UNMANNED AERIAL VEHICLES COMPANY: A STOCHASTIC MODEL OF RESOURCES OVERBOOKING
}

\author{
Mihail Smurov ${ }^{1}$, Nikolajs Sulima ${ }^{2}$ \\ ${ }^{1}$ Saint Petersburg State University of Civil Aviation \\ 38 Pilotov, Saint Petersburg, Russia 196210 \\ +0078127041519,rector@spbguga.ru \\ ${ }^{2}$ La Castelana $S A$ \\ Villa La Jolla 17, A Suneion de Bellu, 325 m at Hose, Costa Rica \\ sulima@gmail.com
}

In connection with the rapid development of commerce of unmanned aerial vehicles (UAV), the optimality of their use with UAV companies (UAVCo) is becoming increasingly important. The basis of such companies are mobile units (mobile units $(\mathrm{MU})$ ), which include UAV, means of their delivery to the place of rendering services and maintenance personnel. A problem of determining the optimal level of orders for the use of MU with UAVCo has been formulated and solved. The task is formulated as an overbooking task. The process under investigation is described as a discrete-time Markov chain corresponding to 24 hours. Based on a fixed number of MU and a fixed overbooking level, distributions for the number of orders on hand, the number of unfulfilled customs, and the average income with an MU usage fee and penalties for unfulfilled orders factored in have been calculated. The solution method has been tested on a particular model. For calculation used language and computer environment MathCAD.

Keywords: unmanned aerial vehicles, overbooking, Markov chain

\section{Introduction}

The improvement of regulations on the use of unmanned aerial vehicles (UAV) in the commercial sphere contributes to their increased use in different sectors of the economy, such as infrastructure, agriculture, transportation, insurance, mass media and entertainment, the mining industry, etc. (Federal Aviation Administration, 2016; Carey London Limited, 2016; Dronelife, 2015; DroneDeploy, 2016). The global market for services provided by UAV companies (UAVCo) is growing rapidly. It exceeded $\$ 130$ billion in 2016, with more than half of this market (61\%) accounted for by infrastructure ( $\$ 45.2$ billion) and agriculture ( $\$ 32.4$ billion).

We will consider UAVCo to be commercial if they:

- provide their services for a fee;

- work on an ongoing basis; activities;

- have all the necessary permits from aviation and other types of authorities for performing their

- employ certified pilots / operators.

UAVCo provide a variety of services connected with aerial photography, as well as detecting and tracking objects, processes, and events.

The main resource of UAVCo is mobile units (MU) that are either located in one particular area or distributed over a number of areas, fulfilling orders placed in their regions. An MU consists of a UAV, a vehicle (usually a specially equipped car) for the delivery of the UAV to the location of the service provision, a drone operator and an assistant (one of them is also the driver of the vehicle). UAVCo resources are reserved with the help of information systems (IS), the way it is done in the field of air transport. However, the structure of UAVCo IS, unlike that of computer reservation systems (CRS) used by airlines, lacks optimization products that could make it possible to implement strategies such as Revenue Management. It is known that the use of these products provides an increase of 3-7 percent in the incomes of traditional airlines, resulting in tens of billions of dollars of additional revenue per year (Cross, 1997; Kaul, 2009; Smith et al., 1992; Rebezova et al., 2012).

One of the methods of the Revenue Management strategy is overbooking (Netessine and Shumsky, 2002; Rothstein, 1971). Overbooking is an effective means of increasing revenues of airlines and other companies providing services (Netessine and Shumsky, 2002; Rothstein, 1971). Overbooking is a marketing policy where, in order to generate additional income, reservations are taken beyond the capacity for accommodation. 
The aim of this study is to develop a stochastic model for optimizing the management of UAVCo revenues based on the overbooking method. In order to achieve this goal, we have identified the features of the overbooking of UAVCo resources, set up a corresponding optimization problem, chosen the solution method, and validated the model using a number of examples.

The overbooking method began to be used in air transport and other services in the $1950 \mathrm{~s}$. At that time, up to 10 percent of passengers who bought airline tickets for flights did not arrive for boarding and later asked for a refund on their tickets. Thus, airlines carried empty seats instead of passengers, regularly experiencing huge losses. The use of the overbooking method allowed airlines quite reasonably to sell more tickets than there were places on the flight on the calculation that some passengers would not arrive for the flight and return their tickets. However, there was a risk that the number of passengers arriving for the flight would be bigger than the number of seats. In this situation, the airline is forced to compensate the customer for the inconvenience caused according to corporate rules (sending the passenger with the next flight offering a higher class of service, providing the passenger with food and accommodation, providing financial compensation, etc.). Thus, the effectiveness of overbooking models is determined by the difference between the gain from the sale of non-existing seats and the losses from the inability of the airline to fulfil its obligations on a particular flight if it is overbooked.

The simplest overbooking models are based on the use of statistical information from the airline's CRS about the distribution (usually normal) of the number of passengers who did not arrive but had guaranteed (i.e. paid) reservations to the number of flights (Netessine and Shumsky, 2002; Rothstein, 1971; Littlewood, 1972).

As a rule, more complex overbooking models used both as part of separate Revenue Management products in such as Amadeus, Galileo, Sabre, and as part of other systems (Phillips, 2005; Belobaba, 2006):

- use the mathematical methods of probability theory and dynamic programming to predict flight load, providing details on booking codes, flights and dates, taking into account interline agreements with other airlines;

- calculate revenue load for the near future and a more distant future, taking into account market conditions.

However, products that use these models cannot be applied to overbooking UAVCo resources, as they are, firstly, excessive in the number of types of overbooking resources (in UAVCo all resources are of the same type), secondly, they are quite expensive (hundreds of thousands of dollars) and, thirdly, calculations can be time-consuming.

The present study develops a model (Sulima, 2012) based on the use of the theory of Markov chains, which optimizes overbooking of only one type of resource (i.e. seats of one booking class, which is suitable for overbooking UAVCo resources of the same type) and differs from the previously mentioned models in that overbooking as a method is used long before the flight departure if all the seats have already been sold. The development of the model (Sulima, 2012) consists in including such factors as force-majeure events (weather conditions and other conditions that make it impossible to use UAV, penalties paid to customers for cancelling orders on the day of their fulfilment).

\section{Model Formulation and Solution}

In order to solve the task of model development, we will use the following notation:

$n$ - the number of MU at the company's disposal;

$\lambda(t)$ - the average number of orders on the day which is $t$ days prior to their fulfilment (in fact, this number has a given integer distribution);

$q$ and $q 0$ - probabilities of an order being withdrawn on the day before its fulfilment and on the day of its fulfilment, respectively (the events are mutually independent for different orders and do not depend on time);

$a$ - income for each MU used during daylight hours;

$b-$ a penalty paid by the client for cancelling the order on the day of its fulfilment or in case of non-fulfilment of the contractual terms for the preparation of the facility for service provision (order cancellation in advance, i.e. more than one day before its fulfilment, is not penalized);

$d$ - losses from compensation to the client for an unfulfilled order as a result of applying the overbooking strategy;

$f$ - a probability that a force majeure event may occur on the day when the order was to be fulfilled.

A stochastic model of resources overbooking will be determined in two steps: 
1) finding the distribution of orders fulfilled $(W)$ and the distribution of orders cancelled $(U)$ on the day of their fulfilment;

2) finding the maximum number of orders accepted $(r(k))$ for each day that is $k$ days prior to order fulfilment in order to maximize the average income.

A few days $(k)$ prior to the day of service provision, the total number of orders received is $m$, so the number of additional orders is $r=\min \{m-n, 0\}$. We would like to find the probability that $i$ MU will be busy on the day of service provision.

The total number of all orders coming on a certain day is a random variable $N$ having a Poisson distribution with parameter $\Lambda$. This means that the average number of orders per day is $\Lambda$. The share of orders placed $k$ days before their fulfilment is $\varphi(k)$. Function $\varphi(k)$ can be calculated based on the statistical data on the number of days that it takes to fulfil orders. As a result, the input flow of orders on the day analysed is nonstationary, with the intensity of $\lambda(k)=\Lambda \varphi(k)$ for the day which is $k$ days prior to order fulfilment. The corresponding distribution will be approximated by a Poisson law with parameter $\lambda(k)$.

Let $X(k)$ denote the number of orders that the company has on the day which is $k$ days prior to their fulfilment, and $p_{i}(k)$ be the corresponding probability: $P\{X(k)=i\}=p_{i}(k)$. Then $X(0)$ is the number of orders at the moment of their fulfilment. If $i>n$, then several orders $(L=i-n)$ will not be fulfilled.

In order to calculate the probabilities $\left\{p_{i}(k)\right\}$, we will connect the distributions of random variables $X(k)$ and $X(k+1)$ for two consecutive days. It is clear that the distribution of $X(k)$ depends only on the value of $X(k+1)$ rather than on the whole chain of $X(\tau), X(\tau-1), \ldots, X(k+1)$, where $\tau$ is the maximum amount of time before the fulfilment of an order. The sequence of $X(\tau), X(\tau-1), \ldots, X(k+1)$ is therefore a Markov chain (Kijima, 1997). Thus, we need to find the conditional probabilities of transition per step (i.e. day) $P\{X(k)=i \mid X(k+1)=j\}$.

If $X(k)=j$, then the number of remaining orders at the end of day $Z$ has a binomial distribution with parameters $j$ and $1-q$. Consequently,

$$
P\{Z=m \mid X(k)=j\}=\frac{j !}{m !(j-m) !}(1-q)^{m} q^{j-m}, m=0, \ldots, j .
$$

The number of new orders on day $Y$ has a Poisson distribution with parameter $\lambda(k)$ :

$$
P\{Y=i\}=\frac{1}{i !} \lambda(k)^{i} \exp (-\lambda(k)), \quad i=0, \ldots, .
$$

The sum of these values gives the total number of orders:

$$
\begin{aligned}
& P\{Z+Y)=i \mid X(k)=j\}=\sum_{m=0}^{i} P\{Z=m \mid X(k)=j\} P\{Y=i-m\}= \\
& =\sum_{m=0}^{i} \frac{j !}{m !(j-m) !}(1-q)^{m} q^{j-m} \frac{1}{(i-m) !} \lambda(k)^{i-m} \exp (-\lambda(k)), i=0, \ldots
\end{aligned}
$$

If the number of orders is greater than $n+r(k)$, no additional orders are accepted.

Consequently, the number of orders at the beginning of the next day has the following conditional distribution for $k=\tau-1, \ldots, 0, i \leq n+r(k)$ :

$$
\begin{aligned}
& p_{i}(k \mid j)=P\{X(k)=i \mid X(k+1)=j\}=\sum_{m=0}^{n+r(k)} P\{Z=m \mid X(k+1)=j\} P\{Y=i-m\}= \\
& =\sum_{m=0}^{n+r(k)} \frac{j !}{m !(j-m) !}(1-q)^{m} q^{j-m} \frac{1}{(i-m) !} \lambda(k)^{i-m} \exp (-\lambda(k)), i=0, \ldots, n+r(k)-1 . \\
& p_{i}(n+r(k) \mid j)=P\{X(k)=i \mid X(k+1)=j\}=\sum_{m=n+r(k)}^{\infty} P\{Z=m \mid X(k+1)=j\} P\{Y=i-m\}= \\
& =\sum_{m=n+r(k)}^{\infty} \frac{j !}{m !(j-m) !}(1-q)^{m} q^{j-m} \frac{1}{(i-m) !} \lambda(k)^{i-m} \exp (-\lambda(k)) .
\end{aligned}
$$


Here $X(\tau)$ has a specified value.

For $k=\tau-1, \ldots, 0$, the unconditional distribution can be described as:

$p_{i}(k)=P\{X(k)=i\}=\sum_{j=0}^{n+r(k)} P\{X(k+1)=j\} p_{i}(k \mid j), \quad i=0, \ldots, n+r(k)$.

Equation (4) allows us to find the distribution of the number of orders on any day before their fulfilment. Calculations should start from the most remote day $\tau$, for which the number of available orders

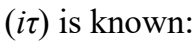

$$
P\{X(\tau)=i \tau\}=1 \text {. }
$$

Next, we should do calculations for each consecutive day moving closer to the departure day. The end point is the day of order fulfilment, for which $k=0$. The number of orders at the beginning of this day is $X(0)$.

Now we should take orders cancelled on the final day into consideration. With $j$ initial orders, the number of one remaining after cancellations has a binomial distribution with parameters $j$ and $q 0$ :

$$
P\{X=i \mid X(0)=j\}=\frac{j !}{i !(j-i) !}(1-q 0)^{i} q 0^{j-i}, i=0, \ldots, j .
$$

The unconditional distribution of $X$ orders at the moment of their fulfilment can be described as:

$$
P\{X=i\}=\sum_{j=i}^{n+r(0)} P\{X(0)=j\} P\{X=i \mid X(0)=j\}, i=0, \ldots, n+r(0) .
$$

The final distribution of the number of orders fulfilled $(W)$ is obtained from the following expressions:

$$
\begin{aligned}
& P\{W=i\}=\sum_{j=i}^{n+r(0)} P\{X(0)=j\} P\{X=i \mid X(0)=j\}= \\
& =\sum_{j=i}^{n+r(0)} p_{j}(k) \frac{j !}{i !(j-i) !}(1-q 0)^{i} q 0^{j-i}, i=0, \ldots, n-1 \\
& P\{W=n\}=\sum_{j=n}^{n+r(0)} p_{j}(k) \sum_{i=n}^{n+r(0)} \frac{j !}{i !(j-i) !}(1-q 0)^{i} q 0^{j-i} .
\end{aligned}
$$

The distribution of the number of orders cancelled on the day of their fulfilment $(U)$ is obtained from the following expression:

$$
\begin{aligned}
& \left.P\{U=i\}=\sum_{j=n+1}^{n+r(0)} P\{X(0)=j\} P\{X=i+n) \mid X(0)=j\right\}= \\
& =\sum_{j=n+i}^{n+r(0)} p_{j}(k) \frac{j !}{(i+n) !(j-(i+n)) !}(1-q 0)^{i+n} q 0^{j-(i+n)}, \quad i=0, \ldots, r(0) .
\end{aligned}
$$

\section{Formulation of the Optimization Criterion}

Let us consider the following groups of orders on a particular day:

1) All orders at the beginning of the final day. The corresponding distribution of their number $(X(0))$ can be obtained from equations (1) - (4).

2) Orders remaining after cancellations of orders in the final day. The distribution of this random variable $\left(p_{i}(k)\right)$ can be obtained from equations (6) and (7). The number of orders cancelled on the final day is $X(0)-X$.

3) Orders fulfilled per day $(W)$. The corresponding distribution can be obtained from expression (8).

4) Orders unfulfilled. The distribution of this random variable $(U)$ can be obtained from expression (9). 
The average values of the random variables mentioned above can be obtained from the following equations:

$$
\begin{aligned}
& E(X(0))=\sum_{m=0}^{n+r(k)} i \times P\{X(0)=i\} . \\
& \left.E(X \mid X(0)=j)=\sum_{i=0}^{j} i \times P\{X=i \mid X(0)=j\}\right) . \\
& E(W)=\sum_{i=0}^{n} i \times P\{W=i\} . \\
& E(U)=P\{U=i\}=\sum_{i=0}^{r(0)} i \times P\{U=i\} .
\end{aligned}
$$

The average income $(C)$, as an optimization criterion, is calculated as follows:

$$
C=\max \{a E(W)+b[E(X(0)-E(X)]-d E(U)\} .
$$

Now the task is to specify the maximum number of orders $r(k)$ for each $k$ days prior to their fulfilment, that would maximize the average income (11).

\section{Computational Aspects and a Numerical Example}

The question of overbooking arises when the number of orders received approaches, reaches or exceeds the number of MU at the company's disposal. We will therefore take the point when there are $\tau$ days left before order fulfilment as the beginning of the process. Let us assume that $\tau$ is a viewing horizon. Let there be $X(\tau)=i \tau$ orders at this moment. It is from these values, the sequence of iterations starts (expression (1) - (4)).

The next remark concerns the amount of data to be stored. If the number of drones is $n$ and the maximum resale value is $r=\max \{r(k): k=\tau, \ldots, 0\}$, then the number of orders received cannot exceed $n+r$. Consequently, the number of possible values of random variables $X(k), X, U$ and $W$ does not exceed $n+r+1$. Therefore, for example, the distribution of $X(k)$ for all $\tau+1$ days will require the storage of only $(\tau+1)(n+r+1)$ numbers.

Let $X(k)$ denote the number of orders $k$ days prior to their fulfilment, with $p_{i}(k)$ denoting the corresponding probability: $P\{X(k)=i\}=p_{i}(k)$.

We will use the following initial data:

- $\quad n=10 ; \Lambda=8 ; a=100 ; b=20 ; d=30 ; f=0 ; q=0.02$ and $q 0=0.05$;

- the probability distribution $\varphi(t)$ of order time $T$ prior its fulfilment is binomial with parameters $p=0.5$ and $\tau=10$. The average of this time is $E(T)=p \tau=0.5 \times 10=5$, and the corresponding variance is calculated as $D(T)=p(1-p) \tau=0.5 \times 0.5 \times 10=2.5$;

- quotas for all extra orders coincide, i.e. $r(k)=r=2, k=\tau, \ldots, 0$.

Table 1 shows the distributions of the random variables of the number of orders received $(X(k)) k$ days before their fulfilment which were calculated successively using equations (1) - (4) for the initial condition $X(\tau)=1$.

Table 1. The distributions $\left\{p_{i}(k)\right\}$ of random variables $X(k)$

\begin{tabular}{|l|l|l|l|l|l|l|l|l|l|}
\hline $\mathbf{k}$ & $\boldsymbol{\tau}=\mathbf{8}$ & $\boldsymbol{\tau}=\mathbf{7}$ & $\boldsymbol{\tau}=\mathbf{6}$ & $\boldsymbol{\tau}=\mathbf{5}$ & $\boldsymbol{\tau}=\mathbf{4}$ & $\boldsymbol{\tau}=\mathbf{3}$ & $\boldsymbol{\tau}=\mathbf{2}$ & $\boldsymbol{\tau}=\mathbf{1}$ & $\boldsymbol{\tau}=\mathbf{0}$ \\
\hline $\mathrm{i}=0$ & 0 & .016 & .013 & .003 & .000 & .000 & .000 & .000 \\
\hline $\mathrm{i}=1$ & 1 & .767 & .328 & .064 & .009 & .003 & .001 & .000 \\
\hline $\mathrm{i}=2$ & 0 & .191 & .359 & .169 & .038 & .011 & .006 & .006 & .001 \\
\hline $\mathrm{i}=3$ & 0 & .024 & .200 & .234 & .0090 & .032 & .020 & .019 & .006 \\
\hline $\mathrm{i}=4$ & 0 & .0002 & .074 & .219 & .144 & .068 & .046 & .044 & .048 \\
\hline $\mathrm{i}=5$ & 0 & .000 & .021 & .155 & .176 & .110 & .082 & .079 & .083 \\
\hline $\mathrm{i}=6$ & 0 & .000 & .005 & .088 & .173 & .142 & .117 & .114 & .119 \\
\hline $\mathrm{i}=7$ & 0 & .000 & .001 & .041 & .142 & .155 & .141 & .139 & .142 \\
\hline $\mathrm{i}=8$ & 0 & .000 & .001 & .017 & .100 & .145 & .146 & .145 \\
\hline $\mathrm{i}=9$ & 0 & .000 & .000 & .006 & .062 & .119 & .133 & .134 & .146 \\
\hline $\mathrm{i}=10$ & 0 & .000 & .000 & .002 & .034 & .087 & .108 & .112 & .111 \\
\hline $\mathrm{i}=11$ & 0 & .000 & .000 & .001 & .017 & .058 & .082 & .093 \\
\hline $\mathrm{i}=12$ & 0 & .000 & .000 & .000 & .013 & .071 & .117 & .115 \\
\hline
\end{tabular}


The distributions of the number of orders $X\{P\{X=i\}\}$ and the number of orders fulfilled $W$ $\{P\{W=i\}\}$ are shown in Figure 1, and the distribution of unfulfilled orders $U\{P\{U=i\}\}$ is shown in Figure 2.

The average values of indicators $E(X(0)), E(X), E(W))$ are presented in Figure 3, and those of $E(U)$ and $C$ are shown in Figures 4 and 5 respectively. These values have been calculated based on the previous conditions with the level of overbooking $r=0,1, \ldots 6$. The data in Figure 5 show that the maximum average income $C=747.826$ can be obtained with $r=2$.

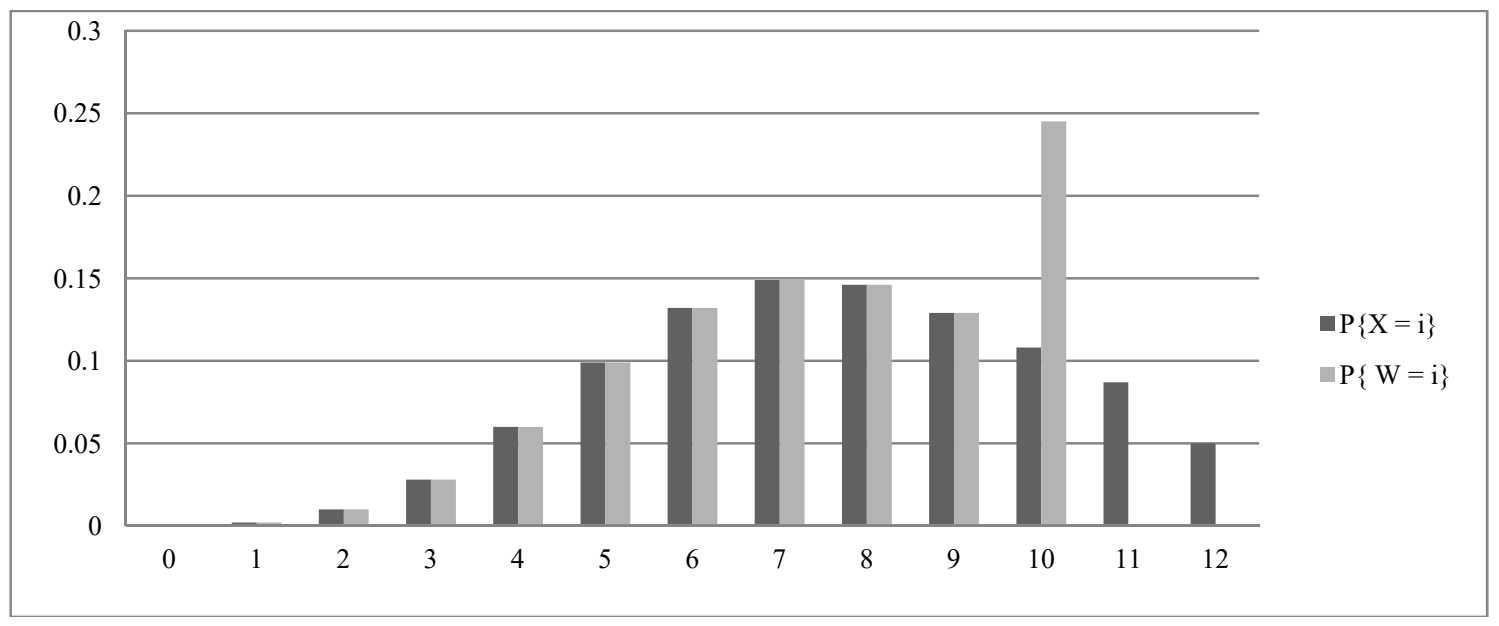

Figure 1. Distributions $\{P\{X=i\}\}$ and $\{P\{W=i\}\}$ of $X$ and $W$

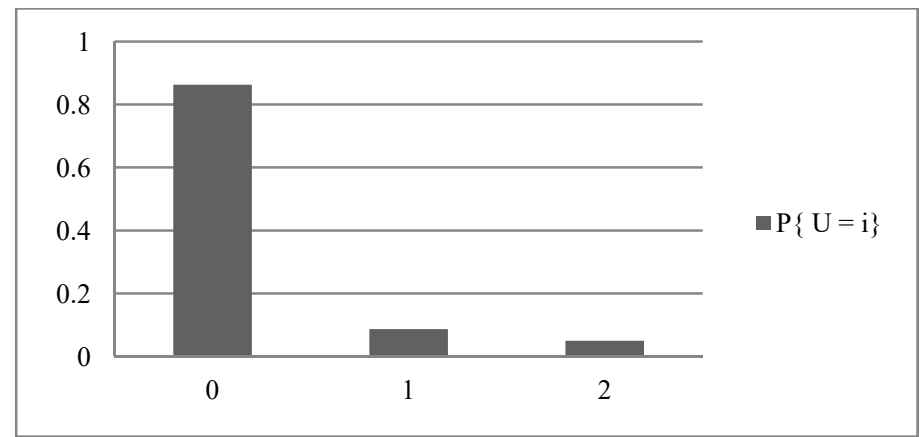

Figure 2. Distribution of unfulfilled orders $U$

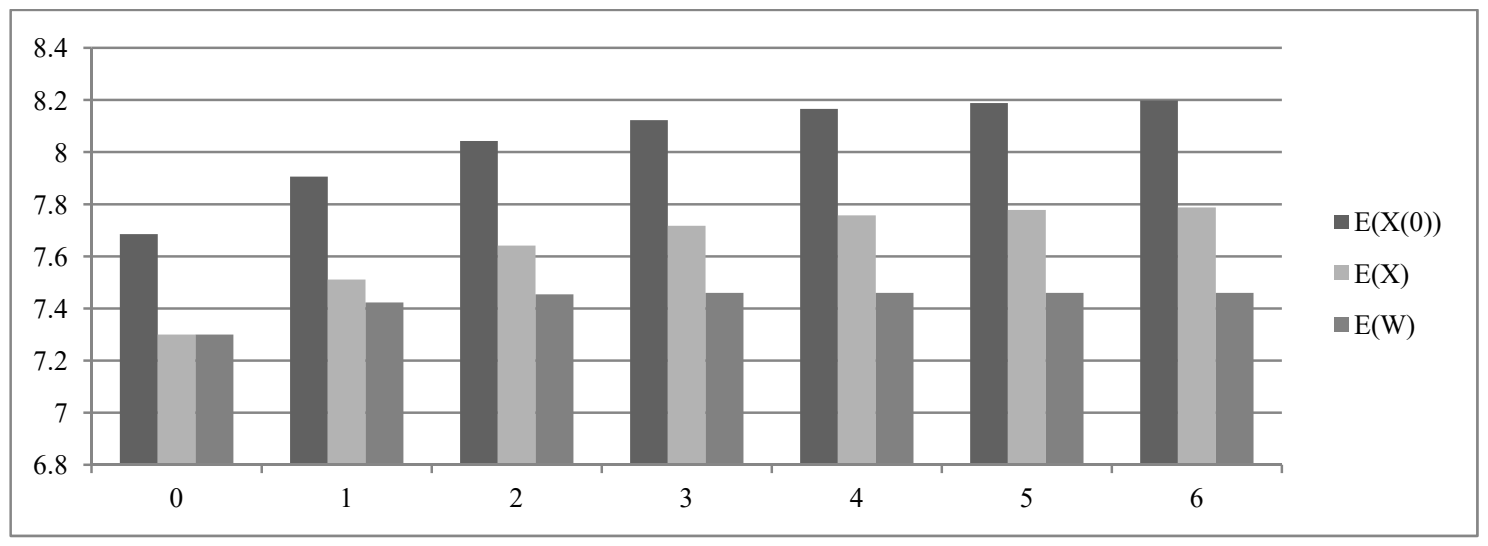

Figure 3. The average values of $E(X(0)), E(X), E(W)$ as a function of $r$ 


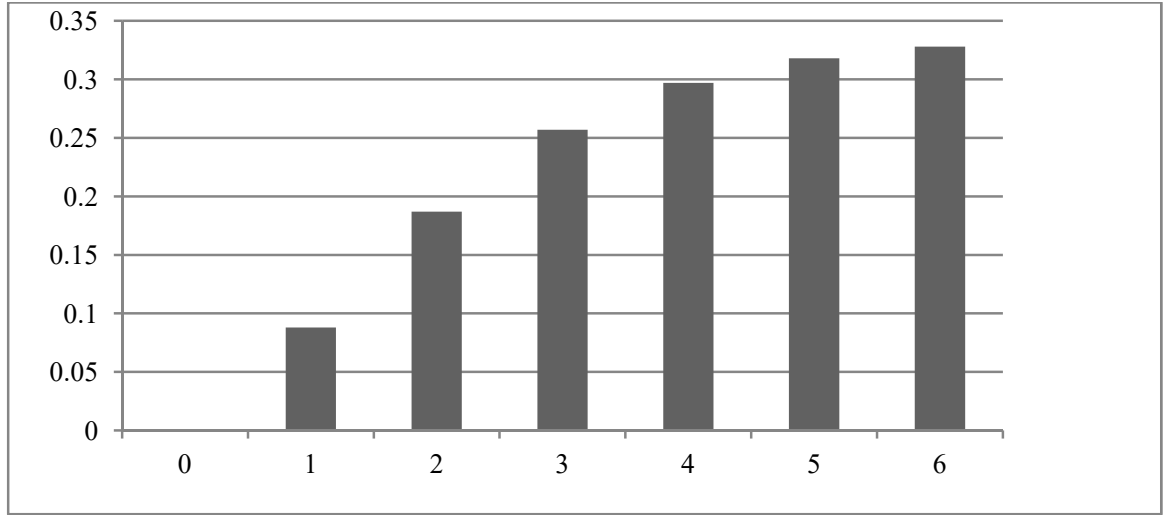

Figure 4. The average values of $E(U)$ as a function of $r$

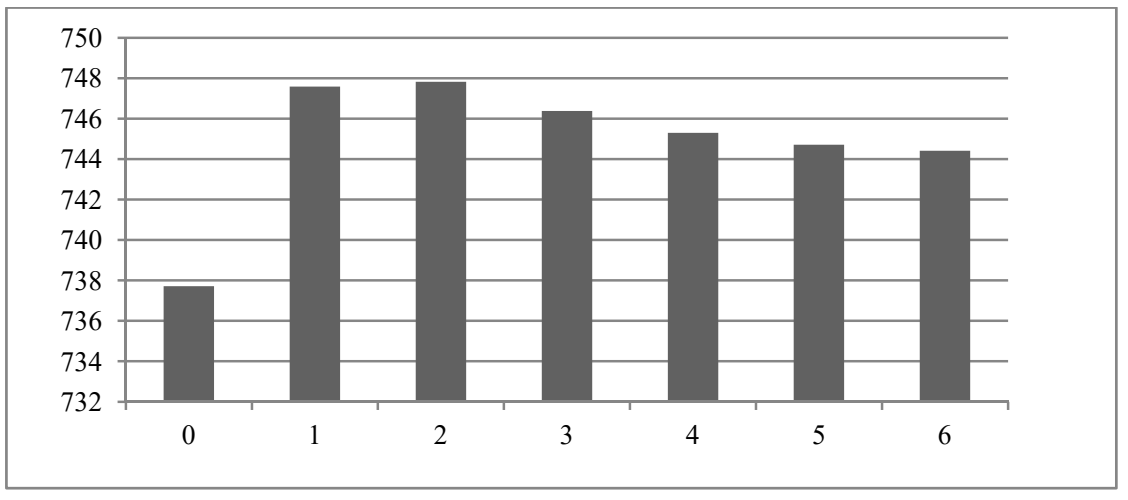

Figure 5. The average values of $C$ as a function of $r$

Let us develop the example further. Earlier it is supposed that number $n$ of MU is fixed and equals 10. Let us find the optimal number $n$ that would yield the highest average income $C$ under the initial conditions given above and with the following additional data:

- the cost of maintaining one MU per day equals 10 units;

- the level of overbooking is $20 \%$ of the number of MU, i.e. $r=0.2 n$ rounded to the nearest whole number.

The results of calculations for $n$ ranging from 5 to 14 show (Fig. 6) that the optimal number of MU equals 12, and the optimal level of overbooking equals 2 .

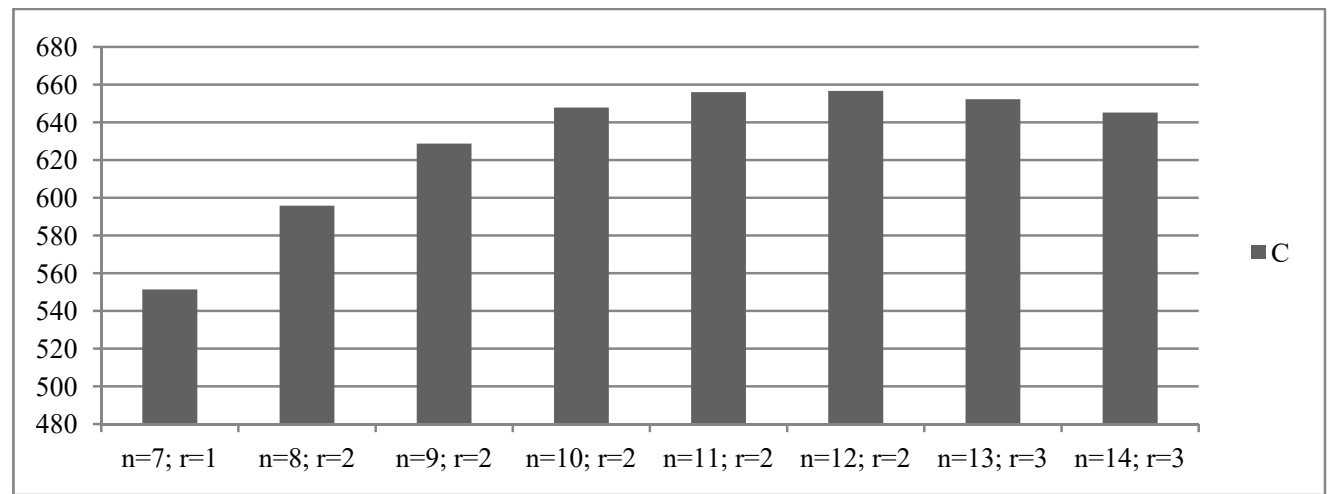

Figure 6. A column chart indicating the optimal values of $n$ and $r$ yielding the highest value of $C$

Note that for $r=2$ the column representing $n=10$ (Fig. 6) differs from the corresponding column in Figure 5 by 100, which is the daily cost of maintenance of $10 \mathrm{MU}$. 


\section{Conclusions and Future Research Directions}

The problem of analyzing overbooking of orders for the use of MU as part of UAVCo has been formulated and solved. The numerical example illustrates the suggested approach. It is supposed in the example that the order level has a constant value and does not depend on the time remaining till its fulfilment.

\section{References}

1. Belobaba, P.P. (2006) Flight Overbooking: Models and Practice 16.75J/1.234J Airline Management. - https://ocw.mit.edu/courses/aeronautics-and-astronautics/16-75j-airline-managementspring-2006/lecture-notes/lect19.pdf/ Retrieved 10 November 2016.

2. Carey London Limited: The Eye in the Sky: How Drones are Transforming the Construction Industry - $\quad$ https://careylondonltd.co.uk/eye-sky-drones-transforming-construction-industry/. Retrieved 10 May 2016.

3. Cross, R. (1997) Revenue Management: Hard-Core Tactics for Market Domination. New York: Broadway Books.

4. DroneDeploy: How to price your drone mapping services - https://blog.dronedeploy.com/how-toprice-your-drone-mapping-services-b5fc31c86934/. Retrieved 10 November 2016

5. Dronelife: 7 advice aerial photography company - http://dronelife.com/2015/05/12/7-advice-aerialphotography-company/. Retrieved 10 November 2016.

6. Federal Aviation Administration: Drones in America Must Now Be Registered. Here's What You Need to Know. Inc. - https://www.faa.gov/uas/media/Part_107_Summary.pdf. Retrieved 10 May 2016.

7. Kaul, S. (2009) Yield Management: Getting More Out of What You Already Have, Ericsson Business Review No. 2: 17-19.

8. Kijima, M. (1997) Markov Processes for Stochastic Modelling. London: Chapman \& Hall.

9. Littlewood, K. (1972) Forecasting and Control of Passenger Bookings. AGIFORS Symposium Proceedings. 12, 96-117.

10. Netessine, S., Shumsky, R. (2002) Introduction to the Theory and Practice of Yield Management. INFORM Transactions on Education, Vol. 3, No. 1.

11. Phillips, R.L. (2005) Pricing and revenue optimization. Stanford University Press. 355 p. ISBN 9780804746984

12. Rebezova, M., Sulima, N., Surinov, R. (2012) Development Trends of Air Transport Services and Service Distribution Channels. Riga: Transport and Telecommunication, 13 (2), 159-166.

13. Rothstein, M. (1971) An Airline Overbooking Model. University of Connecticut, Storrs, Connecticut. Permalink: - http://dx.doi.org/10.1287/trsc.5.2.180 Published Online: May 1, 1971 Page Range: 180-192.

14. Smith, B.C., Leimkuhler, J.F. and Darrow, R.M. (1992) Yield Management at American Airlines. Interfaces. Vol.22, pp. 8-31.

15. Sulima, N. (2012) Probabilistic Model of Overbooking for an Airline. Automatic Control and Computer Sciences, (C) Allerton Press Inc. 46(1), pp. 49-56. 